\title{
Perfil e fatores associados ao consumo alcoólico entre acadêmicos da área de saúde de uma universidade de Montes Claros, Minas Gerais, Brasil
}

\author{
Profile and factors associated with alcohol consumption among health students at a \\ university of Montes Claros, Minas Gerais, Brazil \\ Perfil y factores asociados al consumo alcohólico entre estudiantes de salud en una \\ universidad de Montes Claros, Minas Gerais, Brasil
}

Fabíola Afonso Fagundes Pereira ${ }^{1 *}$, Andra Aparecida da Silva Dionízio ${ }^{1}$, Danilo Cangussu Mendes ${ }^{1}$, Deborah Fernanda Nunes Moreira ${ }^{1}$, Karinne Lima Serrat ${ }^{1}$, Karyne Gabriele Leite Alves ${ }^{1}$, Julia Maria Gonçalves de Almeida ${ }^{1}$.

\section{RESUMO}

Objetivo: Conhecer o perfil e os fatores associados ao uso de bebidas alcoólicas, entre acadêmicos da área da saúde de uma universidade de Montes Claros, Minas Gerais. Métodos: Trata-se de um estudo transversal e analítico, realizado com 331 estudantes matriculados nos cursos da área da saúde de uma universidade do norte de Minas Gerais. Foi aplicado o questionário Alcohol Use Disorders Identification Test (AUDIT) acrescido de perguntas sociodemográficas adaptadas para este estudo. Realizou-se análise descritiva (Teste qui quadrado de Pearson) e a Regressão logística binária, aplicando a técnica stepwise backward. Resultados: O estudo mostrou que $52,3 \%$ dos acadêmicos tinham o hábito de ingerir bebidas alcoólicas e $79,5 \%$ dos entrevistados apresentaram um baixo risco para o consumo de álcool ou são abstêmios. $E$ os indivíduos que apresentaram maiores chances de consumo de risco foram: ter amigos que bebem $(O R=22,826$; valor $p=0,002)$, ter filhos $(O R=4,757$; valor $p=0,025)$, ter algum tipo de trabalho formal ou informal $(O R=4,624$; valor $p=0,013)$, ser solteiro $(O R=10,184$; valor $p=0,02)$ e ter familiares que bebem ( $O R=1,983$; valor $p=0,042)$. Conclusão: Constatou-se assim, a necessidade de ações educativas para conscientização dos universitários acerca do consumo de bebidas alcoólicas e das consequências do uso abusivo.

Palavras-chave: Estudantes de ciências da saúde, Consumo de álcool na faculdade, Alcoolismo, Estudos transversais.

\begin{abstract}
Objective: To know the profile and factors associated with alcohol consumption among health students of a university in Montes Claros, Minas Gerais. Methods: This is a quantitative, cross-sectional and analytical study conducted with 331 students enrolled in one of five health courses at a university in northern Minas Gerais. It was applying the Alcohol Use Disorders Identification Test questionnaire (AUDIT) plus sociodemographic questions adapted for this study. Descriptive analysis, Pearson's chi-square test) and binary logistic regression were performed by applying the stepwise backward technique. Results: The study showed that $52.3 \%$ of the students had the habit of drinking alcohol and $79.5 \%$ of respondents had a low risk for alcohol consumption or are abstainers. And the individuals who had the highest chances of risky consumption were: having friends who drink $(O R=22.826 ; p=0.002)$, having children $(O R=4.757 ; p=0.025)$, having some type of formal or informal work $(O R=4.624 ; p=0.013)$, being single $(O R=10.184 ; p=0.02)$. Conclusion: Thus, it was found the need for educational actions to raise awareness among undergraduates about the consumption of alcohol and the consequences of abuse.
\end{abstract}

Keywords: Students health occupations, Alcohol drinking in college, Alcoholism, Cross-sectional studies.

${ }^{1}$ Universidade Estadual de Montes Claros, Montes Claros - MG. *E-mail: fa_fagundesp@yahoo.com.br 


\section{RESUMEN}

Objetivo: Conocer el perfil y los factores asociados con el consumo de alcohol entre estudiantes de salud de una universidad en Montes Claros, Minas Gerais. Métodos: Este es un estudio cuantitativo, transversal y analítico realizado con 331 estudiantes matriculados en uno de los cinco cursos de salud en una universidad en el norte de Minas Gerais. Estaba aplicando el cuestionario Prueba de Identificación de Trastornos por Consumo de Alcohol (AUDIT) más preguntas sociodemográficas adaptadas para este estudio. El análisis descriptivo, la prueba de chi-cuadrado de Pearson) y la regresión logística binaria se realizaron aplicando la técnica de retroceso gradual. Resultados: El estudio mostró que el $52.3 \%$ de los estudiantes tenían el hábito de beber alcohol y el $79.5 \%$ de los encuestados tenían un bajo riesgo de consumo de alcohol o se abstienen. $\mathrm{Y}$ las personas que tenían las mayores posibilidades de consumo de riesgo fueron: tener amigos que beben $(O R=22.826 ; p=0.002)$, tener hijos $(O R=4.757 ; p=0.025)$, tener algún tipo de trabajo formal o informal $(O R=4.624 ; p=0.013)$, siendo soltero $(O R=10.184 ; p=0.02)$. Conclusión: Por lo tanto, se encontró la necesidad de acciones educativas para crear conciencia entre los estudiantes universitarios sobre el consumo de alcohol y las consecuencias del abuso.

Palabras clave: Estudiantes del área de la salud, Consumo de alcohol en la universidad, Alcoholismo, Estudios transversales.

\section{INTRODUÇÃO}

Segundo a Organização Mundial de Saúde (OMS), a região das Américas é a segunda em prevalência de consumo de álcool e, em todo o mundo, mais de um quarto $(26,5 \%)$ destes consumidores estão em idade escolar, entre 15 a 19 anos, sendo também uma prática frequente no meio universitário (OMS, 2018; RIBEIRO RM, 2018).

Seu consumo geralmente tem início na adolescência, entre amigos ou em ambiente familiar com concordância ou aceitação dos pais, tendo em vista estarem sob sua tutela e em um ambiente controlado. Neste período, também de formação de personalidade e que os jovens estão submetidos à constante pressão social quanto a sua escolha profissional, vestibular e maioridade, é que os jovens são expostos a propagandas provenientes de estratégia de marketing e publicidade que tem como finalidade induzir a ingestão de bebidas pelo menos em festas ou ocasiões especiais.

Droga essa classificada como lícita e que através das propagandas evidência serem de fácil acesso, o que propicia sua adesão a rituais sociais, a tal ponto de ser, sob pressão social, uma droga componente de momentos sociais e de lazer (ASSOCIAÇÃO BRASILEIRA DE PSIQUIATRIA, SOCIEDADE BRASILEIRA DE MEDICINA DA FAMÍLIA E COMUNIDADE, 2012).

Dentre as mudanças que ocorrem nesse período de transição estão a adaptação dos jovens que ingressam na universidade e que muitas vezes por residirem longe da família, acabam se afastando do seu círculo de relacionamentos familiares e sociais.

Tais mudanças geram o sentimento de autonomia, a necessidade de formar novas amizades, fazer parte de um novo grupo social, se introduzir ao mundo adulto agora sem o controle dos pais e a possibilidade de obter novas experiências, podem estimular situações de crise que faz com que os jovens busquem alternativas para enfrentarem essas situações.

Sendo assim, aumenta a chance do consumo de novas drogas previamente proibidas e muitas vezes ilícitas assim como o aumento no consumo de drogas que já estejam familiarizados a consumir (MONTEIRO LZ, et al., 2018; PELICIOLI M, et al., 2017).

Em um levantamento nacional sobre o uso de álcool, tabaco e outras drogas com 12.711 universitários de 27 capitais brasileiras identificou que $86,2 \%$ destes referiram uso do álcool em algum momento da vida (ANDRADE AG, et al., 2010). Pelicioli M, et al. (2017), em um estudo sobre o perfil do consumo de álcool e prática do beber pesado episódico entre universitários brasileiros da área da saúde, corrobora o resultado anterior, com uma prevalência do consumo de $85 \%$. 
O uso excessivo de álcool pode acarretar problemas sociais, psíquicos e emocionais e aumento da incidência de violência urbana, interferindo na qualidade e expectativa de vida (SAWICKI WC, et al., 2018). No meio acadêmico, as consequências podem ser evidenciadas pelo baixo desempenho nos estudos causado pelos déficits de memória e dificuldades de aprendizado, alterações no sono incluindo sonolência em sala de aula, envolvimento em situações de violência, problemas psicológicos e comportamentais, incluindo transtornos mentais e comprometimento cognitivo além da progressiva dependência do álcool (TRINDADE BPA, et al., 2018).

Os profissionais de saúde são peças fundamentais na mudança de hábitos da população em relação ao álcool (DUARTE PCAV e FORMIGONI MLOS, 2017). Investigar o abuso de álcool entre acadêmicos da área da saúde subsidiará ações de prevenção do consumo abusivo neste contexto, para que se tornem profissionais efetivos no exercício de seu papel. Este estudo tem por objetivo conhecer o perfil e os fatores associados ao uso de bebidas alcoólicas, entre acadêmicos da área da saúde de uma universidade de Montes Claros, Minas Gerais.

\section{MÉTODOS}

Trata-se de um estudo transversal, com estudantes matriculados nos cursos da área da Saúde de uma universidade do norte de Minas Gerais. Essa universidade oferece cursos em quatro áreas distintas: Humanas, Exatas, Sociais Aplicadas, Biológicas e da Saúde. No Centro de Ciências Biológicas e da Saúde havia um total de 1.846 matriculados no primeiro semestre letivo de 2017.

Para seleção dos participantes da pesquisa adotou-se o cálculo da amostragem aleatória simples, com intervalo de confiança de $95 \%$, erro amostral de $5 \%$ e probabilidade do evento de $50 \%$, obtendo-se uma amostra de 320 estudantes. Foi realizado um sorteio das turmas a serem entrevistadas e participaram todos os que atenderam aos critérios de inclusão, totalizando 331 estudantes dos cursos Medicina, Enfermagem, Odontologia, Educação Física e Ciências Biológicas. A coleta foi realizada entre os meses de março e agosto de 2017.

Os critérios de inclusão foram: estar matriculado na primeira semana do mês de março de 2017, com idade superior a dezoito anos, presentes na sala de aula no momento da coleta de dados e aceitar participar do estudo voluntariamente assinando o Termo de Consentimento Livre e Esclarecido. Foi aplicado um questionário auto administrado, o Alcohol Use Disorders Identification Test (AUDIT), acrescido de perguntas sociodemográficas adaptadas para este estudo (DUARTE PCAV e FORMIGONI MLOS, 2017; BAUMGARTEN LZ, et al., 2012).

O AUDIT é um questionário que avalia o consumo recente de álcool, sintomas de dependência e os problemas relacionados ao uso. Foi desenvolvido pela Organização Mundial de Saúde (OMS) e adaptado e validado para a população brasileira por Méndez EB (1999), e posteriormente por Lima CT, et al. (2005).

Este questionário é composto por dez questões tem um escore que vai de 0 a 4 pontos, sendo que 0 somatório dos valores atribuídos a cada questão gera um escore final que pode variar de zero a 40 pontos. Assim é possível classificar o consumo alcoólico em quatro categorias, as chamadas "zonas de risco", de acordo com o intervalo de pontuação: Zona I (baixo risco) - 0 a 7 pontos, refere-se àqueles que podem se beneficiar com informações sobre consumo do álcool; Zona II (uso de risco) - 8 a 15 pontos, refere-se àqueles que mesmo não estejam apresentando problemas atuais, estão correndo o risco de apresentar, em um futuro próximo, problemas de saúde e de sofrer ou causar ferimentos, violências, problemas legais ou sociais e/ou ter baixo desempenho nos estudos, devido aos episódios de intoxicação aguda.

Estes se beneficiariam de orientações que incluem a educação para o uso de álcool e a proposta de estabelecimento de metas para a abstinência ou a adequação do padrão de beber para dentro dos limites considerados de baixo risco; Zona III (uso nocivo) - 16 a 19 pontos, inclui os que, provavelmente, já apresentam problemas e mantêm uso regular, excedendo limites, e se beneficiariam de educação para o uso de álcool, aconselhamento para a mudança do padrão de beber, da análise dos fatores que contribuem para o beber excessivo e o treinamento de habilidades para lidar com estes fatores.; Zona IV (provável dependência) -20 a 40 pontos, inclui aqueles que são prováveis portadores de síndrome de dependência do 
álcool e deveriam ser encaminhados à avaliação especializada para confirmação diagnóstica e possibilidade de tratamento específico (JAEGER GP, et al., 2018; BARROS MSMR e COSTA LS, 2019).

Porém, o enfoque desta pesquisa é apenas determinar o Padrão de consumo e os fatores associados, não foi abordando prevenção ou qualquer forma de assistência. Entretanto, constou-se no questionário informações indicando aos participantes os locais que poderiam buscar assistência de saúde, caso apresentasse problemas relacionados ao uso.

A variável dependente do estudo foi definida como perfil de consumo alcoólico, sendo que, optou-se por categorizar em "Baixo Risco" os entrevistados que se enquadraram na Zona I e em "Alto Risco" os entrevistados que se incluíam nas Zonas II e III conforme a pontuação obtida no questionário AUDIT. Não houve nenhum entrevistado classificado na Zona IV, ou seja, de provável dependência, portanto, essa Zona não foi incluída nas categorias.

As variáveis independentes incluídas no estudo foram: o período que estava cursando ( $1^{\circ}$ ao $3^{\circ} ; 4^{\circ}$ ao $6^{\circ}$, $7^{\circ}$ ao $9^{\circ}$ ), estado civil (solteiro/divorciado/desquitado; casado/união estável), sexo (feminino; masculino), com quem mora (pais e outros familiares; cônjuge ou união estável; amigos/colegas ou outros universitários; sozinho) Filhos (sim; não), manutenção financeira (atividade acadêmica; trabalho formal/informal; auxílio financeiro dos pais), cursou disciplina que aborda alcoolismo (sim; não), família possui hábito de beber (sim; não), amigos possuem hábito de beber (sim; não).

A análise dos dados foi realizada utilizando-se o programa estatístico SPSS ${ }^{\circledR}$ versão 20.0 para Windows. Inicialmente, foi realizada análise descritiva dos dados, seguido da análise bivariada (teste qui quadrado de Pearson) entre a variável dependente "perfil de consumo alcoólico" e as variáveis independentes para determinar os fatores associados.

A partir desta análise, selecionaram-se para o modelo múltiplo (Regressão logística binária) todas aquelas variáveis que apresentaram $p$ valor menor que 0,25 , restando no modelo múltiplo final somente aquelas estatisticamente significativas $(p<0,05)$, utilizando-se a técnica Stepwise Backward (técnica na qual as variáveis que não apresentaram significância no modelo múltiplo vão sendo retiradas uma de cada vez, até que restem somente as significantes).

O estudo foi conduzido de acordo com a Resolução no 466/12 do Conselho Nacional de Saúde sobre Pesquisas com Seres humanos, após aprovação do projeto pelo Comitê de Ética em Pesquisa da UNIMONTES, parecer № 1.866.765, 14 de dezembro de 2016.

\section{RESULTADOS}

Participaram dessa pesquisa 331 universitários, sendo 49 (14,8\%) do curso de Medicina, 54 (16,3\%) de Odontologia, 47 (14,2\%) de Enfermagem, 65 (19,6\%) de Ciências Biológicas e 116 (35\%) de Educação Física. A maioria era do sexo feminino $(n=222 ; 67,1 \%)$, sendo essa prevalência maior no curso de Enfermagem $(89,4 \%)$.

A média de idades dos acadêmicos foi de $21,56( \pm 2,94)$ anos, com idade mínima de 18 e máxima de 42 anos, solteiros(as) ( $n=310 ; 93,7 \%)$, ainda residiam com os pais ou outros familiares $(n=205 ; 61,9 \%)$ e recebiam auxílio financeiro dos pais $(n=231 ; 69,8 \%)$.

Neste estudo, 58,9\% ( $n=195)$ dos entrevistados relataram que seus familiares consomem bebida alcoólica e $77 \%(n=251)$ que seus amigos mais próximos fazem uso de álcool. Dentre os participantes, $52,3 \%$ ( $n=173)$ tinham o hábito de ingerir bebidas alcoólicas. Quanto à pontuação obtida nos escores referentes às zonas de risco, identificou-se que $79,5 \%$ dos entrevistados se enquadram na Zona I, o que significa que tem um consumo de baixo risco de álcool ou são abstêmios.

Apenas uma minoria dos entrevistados foi identificada na Zona II (16,9\%), como consumo de risco, e na Zona III (3,6\%), como consumo nocivo, caracterizadas por ingerir bebidas alcoólicas em quantidade e frequência acima dos padrões de baixo risco e por já ter apresentado problemas decorrentes do consumo do álcool (Tabela 1). 
Tabela 1 - Perfil do consumo de bebidas alcoólicas entre os universitários dos cincos cursos da área da saúde de uma universidade do norte de Minas Gerais, conforme o AUDIT ( $n=331), 2017$.

$\begin{array}{cc}\text { Perfil de consumo* }^{*} & (\%) \\ \text { Baixo risco } & 79,5 \\ \text { Usuários de risco } & 16,9 \\ \text { Uso nocivo } & 3,6\end{array}$

Legenda: *Obtidos a partir da pontuação gerada do AUDIT. Fonte: Pereira FAF, et al., 2020.

Os dados relativos à análise bivariada entre a variável dependente perfil de consumo alcoólico e as variáveis independentes do estudo encontram-se na Tabela 2. Observou-se que todas as variáveis analisadas apresentaram um $p$ valor $<0,25$, sendo assim, inseridas no modelo multivariado.

Tabela 2 - Perfil do consumo de álcool segundo variáveis sociodemográficas entre os universitários dos cincos cursos da área da saúde de uma universidade do norte de Minas Gerais. Brasil, 2017.

\begin{tabular}{|c|c|c|c|}
\hline \multirow{2}{*}{ Variáveis } & \multicolumn{2}{|c|}{ Audit } & \multirow{2}{*}{ Valor $\mathbf{P}$} \\
\hline & Baixo risco N (\%) & Alto risco $\mathrm{N}(\%)$ & \\
\hline \multicolumn{4}{|l|}{ Curso } \\
\hline Medicina & $39(79,6)$ & $10(20,4)$ & \multirow{5}{*}{0,081} \\
\hline Odontologia & $40(74,1)$ & $14(25,9)$ & \\
\hline Enfermagem & $44(93,6)$ & $3(06,4)$ & \\
\hline Ciências Biológicas & $53(81,5)$ & $12(18,5)$ & \\
\hline Educação Física & $87(75,0)$ & $29(25,0)$ & \\
\hline \multicolumn{4}{|l|}{ Período matriculado } \\
\hline $1^{\circ}$ ao 30 & $72(86,7)$ & $11(13,3)$ & \multirow{3}{*}{0,163} \\
\hline $4^{\circ}$ ao $6^{\circ}$ & $109(77,3)$ & $32(22,7)$ & \\
\hline $7^{\circ}$ ao $9^{\circ}$ & $82(176,6)$ & $25(23,4)$ & \\
\hline \multicolumn{4}{|l|}{ Estado Civil } \\
\hline Solteiro/Divorciado/Desquitado & $247(78,9)$ & $66(21,1)$ & \multirow{2}{*}{0,245} \\
\hline Casado/União estável & $16(88,9)$ & $2(11,1)$ & \\
\hline \multicolumn{4}{|l|}{ Sexo } \\
\hline Feminino & $185(83,3)$ & $37(16,7)$ & \multirow{2}{*}{0,010} \\
\hline Masculino & $78(71,6)$ & $31(28,4)$ & \\
\hline \multicolumn{4}{|l|}{ Com quem mora } \\
\hline Pais e outros familiares & $163(79,5)$ & $42(20,5)$ & \multirow{4}{*}{0,247} \\
\hline Cônjuge ou União estável & $15(88,2)$ & $2(11,8)$ & \\
\hline Amigos(as)/colegas/ universitários/outros & $78(80,4)$ & $19(19,6)$ & \\
\hline Sozinho(a) & $7(58,3)$ & $5(41,7)$ & \\
\hline \multicolumn{4}{|l|}{ Filhos } \\
\hline Sim & $10(58,8)$ & $7(41,2)$ & \multirow{2}{*}{0,039} \\
\hline Não & $253(80,6)$ & $61(19,0)$ & \\
\hline \multicolumn{4}{|l|}{ Manutenção Financeira } \\
\hline Atividade acadêmica & $45(77,6)$ & $13(22,4)$ & \multirow{3}{*}{0,172} \\
\hline Trabalho formal/informal & $23(67,6)$ & $11(32,4)$ & \\
\hline Auxílio financeiro dos pais & $188(81,4)$ & $43(18,6)$ & \\
\hline \multicolumn{4}{|l|}{ Cursou disciplina que aborda alcoolismo } \\
\hline Sim & $138(81,7)$ & $31(18,3)$ & \multirow{2}{*}{0,168} \\
\hline Não & $122(76,7)$ & $37(23,3)$ & \\
\hline \multicolumn{4}{|l|}{ Família possui hábito de beber } \\
\hline Sim & $146(74,9)$ & $49(25,1)$ & \multirow{2}{*}{0,009} \\
\hline Não & $117(86,0)$ & $19(14,0)$ & \\
\hline \multicolumn{4}{|l|}{ Amigos possuem hábito de beber } \\
\hline Sim & $184(73,3)$ & $67(26,7)$ & \multirow{2}{*}{$<0,001$} \\
\hline Não & $69(98,6)$ & $1(1,4)$ & \\
\hline
\end{tabular}

Fonte: Pereira FAF, et al., 2020.

Conforme a análise múltipla (Tabela 3), observou-se significância estatística no modelo final para as variáveis: Ter amigos que bebem, curso, ter filhos, Manutenção financeira, estado civil e ter familiares que 
bebem. Assim sendo, os indivíduos que têm maior chance de apresentarem escore médio ou alto do questionário AUDIT são: ter amigos que bebem $(R C=22,826$; valor $p=0,002)$, ter filhos $(R C=4,757$; valor $p=0,025)$, ter algum tipo de trabalho formal ou informal $(R C=4,624$; valor $p=0,013)$, ser solteiro $(R C=10,184$; valor $p=0,02)$ e ter familiares que bebem $(R C=1,983$; valor $p=0,042)$.

Para a variável Cursos aqueles acadêmicos matriculados no curso de Enfermagem apresentaram uma menor chance de serem classificados no escore médio ou alto para o AUDIT, quando comparados com o curso de Medicina ( $R C=0,177$; valor $p=0,021$ ), sendo considerado fator de proteção.

Tabela 3 - Análise ajustada dos fatores associados ao padrão de consumo de álcool na amostra estudada, 2017.

\begin{tabular}{|c|c|c|c|c|}
\hline \multicolumn{2}{|c|}{ Variáveis } & OR & Valor $\mathbf{P}$ & $\mathrm{IC}_{95 \%}$ \\
\hline \multirow{5}{*}{ Curso } & Medicina & Referência & - & - \\
\hline & Odontologia & 1,21 & 0,710 & $0,45-3,24$ \\
\hline & Enfermagem & 0,18 & 0,021 & $0,04-0,77$ \\
\hline & Ciências Biológicas & 0,78 & 0,642 & $0,27-2,22$ \\
\hline & Educação Física & 1,02 & 0,974 & $0,41-2,53$ \\
\hline \multirow{2}{*}{ Ter filhos } & Não & Referência & - & - \\
\hline & Sim & 4,76 & 0,025 & $1,22-18,58$ \\
\hline \multirow{3}{*}{$\begin{array}{l}\text { Manutenção } \\
\text { Financeira }\end{array}$} & Atividade Acadêmica & Referência & - & - \\
\hline & Trabalho formal/informal & 4,62 & 0,013 & $1,39-15,43$ \\
\hline & $\begin{array}{l}\text { Auxílio financeiro dos } \\
\text { pais }\end{array}$ & 1,00 & 0,996 & $0,47-2,13$ \\
\hline \multirow{2}{*}{ Estado Civil } & Casado & Referência & - & - \\
\hline & Solteiro & 10,18 & 0,020 & $1,44-72,21$ \\
\hline \multirow{2}{*}{$\begin{array}{c}\text { Ter familiares que } \\
\text { bebem }\end{array}$} & Não & Referência & - & - \\
\hline & Sim & 1,98 & 0,042 & $1,02-3,84$ \\
\hline \multirow{2}{*}{$\begin{array}{l}\text { Ter amigos que } \\
\text { bebem }\end{array}$} & Não & Referência & - & - \\
\hline & Sim & 22,83 & 0,002 & $3,00-172,85$ \\
\hline
\end{tabular}

Legenda: OR= Odds Ratios; $I_{95 \%}=$ intervalo de confiança de 95\%. Fonte: Pereira FAF, et al., 2020.

\section{DISCUSSÃO}

O presente estudo evidenciou que 173 (52,3\%) acadêmicos(as) da área da saúde entrevistados(as) tinham o hábito de ingerir bebidas alcoólicas, percentual semelhante aos observados em outros estudos (PELICIOLI M, et al., 2017; BAUMGARTEN LZ, et al., 2012; ABREU TT, et al., 2018; PINHEIRO MA, et al., 2017).

Observou-se que $20,5 \%$ dos universitários apresentavam um consumo de risco (Zona II) ou nocivo (Zona III) à saúde. Nenhum dos entrevistados apresentou consumo de provável dependência (Zona IV). Outros estudos apontam um percentual variável de 10,8 até $64,2 \%$ de uso problemático entre universitários que consomem bebidas alcoólicas (PELICIOLI M, et al., 2017; BAUMGARTEN LZ, et al., 2012; CASTAÑO-PEREZ GA e CALDERON-VALLEJO GA, 2014; GUIMARÃES MR, et al., 2017). Resultados como provável dependência foram identificados em $4,2 \%$ dos universitários de uma instituição pública no Estado do Piauí, Brasil e em 14,9\% dos estudantes universitários em Medellín, Colômbia (GUIMARÃES MR, et al., 2017; CASTAÑO-PEREZ GA e CALDERON-VALLEJO GA, 2014).

O uso abusivo de álcool tem como consequências, além do comprometimento das atividades acadêmicas, o envolvimento em situações de violência, acidentes de trânsito, problemas físicos e de relacionamento, dependência e o não uso de preservativos nas relações sexuais que pode levar a gravidez indesejada e infecções sexualmente transmissíveis (BEDENDO A, et al., 2017).

Neste estudo, houve fator de proteção para consumo de risco ou nocivo à saúde aqueles acadêmicos matriculados no curso de Enfermagem quando comparados com os matriculados no curso de Medicina $(p=0,021)$. 
Sobre o consumo Alcóolico entre acadêmicos de Enfermagem, Fernandes MA, et al., (2019) encontrou consumo de risco em $44 \%$ dos participantes, sendo que $58,87 \%$ referiu uso esporádico. Mas o resultado de maior fator de proteção entre os acadêmicos da Enfermagem pode estar associado à prevalência do sexo feminino entre os participantes desse curso $(89,4 \%)$. Vários estudos demonstram forte associação entre 0 consumo nocivo de álcool e estudantes do sexo masculino (BEBENDO A, et al., 2017; RIBEIRO RM, 2018; TRINDADE BPA et al, 2018).

Ribeiro RM (2018), por exemplo, em seu estudo sobre estratégias protetoras e consequências negativas no consumo de bebidas alcoólicas por parte de estudantes universitários, encontrou média superior de estratégias protetoras entre as mulheres e de consequências negativas entre os homens.

Estudo realizado em Passo Fundo, no sul do Brasil, considerando 12 cursos da área da saúde, apontou frequência alta de consumo de bebidas alcoólicas e semelhante entre os cursos de Enfermagem, Farmácia e Medicina. Destes, o curso de Medicina foi o que teve mais estudantes com comportamento de risco em relação ao consumo de bebidas alcoólicas (PELICIOLI M, et al., 2017). Porém, estudo realizado com universitários em geral de uma Universidade do Piauí identificou consumo nocivo, consumo de provável dependência (Zonas III e IV) e maior chance de consumo danoso ao corpo humano em universitários das áreas de Exatas e Humanas (GUIMARÃES MR, et al., 2017).

Neste estudo, os estudantes que se encontravam nos períodos iniciais ( $1^{\circ}$ ao $3^{\circ}$ período) do curso apresentaram o menor índice de consumo alcoólico de alto risco em comparação com os períodos subsequentes, mas não permaneceu estatisticamente significativo na análise múltipla. Já o estudo realizado em uma universidade de São Paulo e outro realizado em Minas Gerais teve associação significativa o aumento no consumo à medida que avançam os períodos (SAWICKI WC, et al., 2018; ABREU TT, et al., 2018).

Entretanto, ter cursado alguma disciplina que aborda o tema alcoolismo não foi significativo para influenciar o consumo alcoólico entre os entrevistados, o que vai de encontro ao observado por outros autores que discute que o conhecimento adquirido no decorrer dos anos de curso não influencia positivamente no controle dos estudantes frente ao consumo de álcool, já que o índice de consumo dessa população encontra-se similar ou maior que os de outros grupos sociais (BAUMGARTEN LZ et al., 2012).

Portanto, o conhecimento acerca do consumo abusivo do álcool e suas consequências não é bem incorporado pelos estudantes, não obstante, eles se sentem aceitos socialmente, influenciados pelas propagandas (ABREU TT, et al., 2018).

Dentre os fatores associados ao consumo Alcóolico, a influência de amigos e familiares configura um dos mais importantes, tanto para a iniciação ao uso do álcool quanto para sua manutenção (FERRAZ L, et al., 2017; GARCIA LP e FREITAS LRS, 2013). Sendo confirmado neste estudo, que o fato de conviver com amigos que bebem aumentam em mais de 20 vezes as chances de ter consumo alcoólico de risco ou nocivo à saúde, e em quase duas vezes quando se tem familiares que bebem.

Estudo realizado numa universidade pública da região Sudeste do Brasil observou que, muitas vezes, o álcool é inserido na vida dos jovens em idade precoce através da família e dos amigos (CAMARGO ECP, et al., 2019). Um estudo da Universidade Federal de São Paulo encontrou que, 90\% dos universitários fizeram uso de álcool alguma vez na vida, e que a idade média de primeiro uso foi de 15,4 anos.

Destes estudantes, 62,8\% referiram estar com amigos no primeiro uso, e 38,3\%, com parentes (SAWICKI WC et al., 2018). Os universitários que tinham um ou mais filhos apresentaram uma maior probabilidade de consumo nocivo se comparados com os universitários que não tinham filhos $(\mathrm{OR}=4,76)$. Contudo, associação entre essa variável "ter filho" e o consumo nocivo não foi significante em outros estudos com esta temática (BAUMGARTEN LZ et al., 2012; ZEFERINO MT, et al., 2015; BETANCOURTH-ZAMBRANO S, et al., 2017).

Relacionando o estado civil ao consumo de alto risco, os solteiros têm 10,18 vezes mais chance de consumo de alto risco. Zeferino MT, et al., (2015), num estudo realizado em Florianópolis, apontam que os estudantes que possuem algum tipo de união afetiva possuem menores probabilidades de consumir drogas lícitas como o álcool. Quanto a variável manutenção financeira, os universitários que exerciam algum tipo de trabalho formal ou informal apresentaram chance 4,62 vezes maior de consumo de alto risco. A independência 
financeira pode contribuir para uma vida social mais ativa, frequentando lugares com maior oferta de bebidas alcoólicas.

Um estudo identificou maiores escores obtidos no perfil do consumo decorrente da escala AUDIT em graduandos da área da saúde com o hábito de frequentar festas uma ou mais vezes por semana (PELICIOLI $M$, et al., 2017). As limitações deste estudo estão relacionadas a definição da amostra, visto que não foi realizado sorteio aleatório de cada participante e sim das turmas a serem entrevistadas.

\section{CONCLUSÃO}

Este estudo mostrou que mais da metade dos universitários da área da saúde pesquisados consomem bebidas alcoólicas, sendo que $20,5 \%$ destes fazem consumo de risco ou nocivo à saúde. E a partir da análise multivariada, observaram-se maiores chances de consumo de risco entre os acadêmicos que tinham amigos e ou familiares que bebem, tinham algum tipo de trabalho formal ou informal, possuíam filhos ou eram solteiros. Entretanto, não é possível generalizar estes resultados para outros universitários e instituições, uma vez que se tratou de uma pesquisa local, sendo necessário maior aprofundamento sobre o assunto com a realização de novos estudos com populações semelhantes. Contudo, percebe-se uma necessidade urgente de ações educativas dentro das universidades que sejam eficazes na conscientização destes acerca do consumo de bebidas alcoólicas e das consequências, principalmente, do uso abusivo, bem como na redução do consumo alcoólico e dos fatores que os encorajam a fazer uso.

\section{REFERÊNCIAS}

1. ABREU TT, et al. O consumo de bebida alcoólica e o binge drink entre os graduandos de medicina de uma universidade de Minas Gerais. J Bras Psiquiatr [Internet], 2018; 67(2): 87-93.

2. ANDRADE AG, et al. I Levantamento nacional sobre o uso de álcool, tabaco e outras drogas entre universitários das 27 capitais brasileiras [Internet]. Brasília; Secretaria Nacional de Políticas sobre Drogas - SENAD/GREA/IPQHC/FMUSP, 2010; $282 \mathrm{p}$.

3. ASSOCIAÇÃO BRASILEIRA DE PSIQUIATRIA, SOCIEDADE BRASILEIRA DE MEDICINA DA FAMÍLIA E COMUNIDADE. Projeto diretrizes: abuso e dependência do álcool. São Paulo, 2012.

4. BARROS MSMR, COSTA LS. Perfil do consumo de álcool entre estudantes universitários Rev. Eletrônica Saúde Mental Álcool Drog, 2019 Jan-Mar; 15(1): 4-13.

5. BAUMGARTEN LZ, et al. Consumo alcoólico entre universitários(as) da área da saúde da Universidade Federal do Rio Grande/RS: subsídios para enfermagem. Esc. Anna Nery [Internet], 2012 set; 16(3): 530-535.

6. BEDENDO A, et al. Binge drinking: padrão associado ao risco de problemas do uso de álcool entre universitários. Rev. Latino-Am. Enfermagem, 2017; 25: e2925.

7. BETANCOURTH-ZAMBRANO S, et al. Consumo de alcohol en estudiantes universitarios colombianos. Rev. Univ. Salud [Internet], 2017; 19(1): 37-50.

8. CAMARGO ECP, et al. Uso e abuso de drogas entre universitários e a sua interface com as políticas públicas SMAD, Rev Eletrônica Saúde Mental Álcool Drog, 2019; 15(4): 1-9.

9. CASTAÑO-PEREZ GA, CALDERON-VALLEJO GA. Problemas associados ao consumo de álcool em estudantes universitários. Rev. Latino-Am. Enfermagem [Internet], 2014; 22(5): 739-46.

10. DUARTE PCAV, FORMIGONI MLOS, organizadoras. Detecção do uso e diagnóstico da dependência de substâncias psicoativas: módulo 3 [Internet]. 11.ed. Brasília: Secretaria Nacional de Políticas sobre Drogas, 2017.

11. FERRAZ L, et al. O uso de álcool e tabaco entre acadêmicos de uma universidade do Sul do Brasil. Rev. Bras. Promoç. Saúde [Internet], 2017; 30(1): 79-85.

12. GARCIA LP, FREITAS LRS. Consumo abusivo de álcool no Brasil: resultados da pesquisa nacional de saúde 2013. Epidemiol. Serv. Saúde [Internet], 2015; 24(2): 227-237.

13. GUIMARÃES MR, et al. Estilo de vida e fatores associados entre estudantes universitários. Rev. Enferm. UFPE on line, Recife, 2017 ago; 11(Supl. 8): 3228-35.

14. JAEGER GP, et al. Transtornos relacionados ao uso de álcool e fatores associados em zona rural do Brasil Rev Saude Publica. 2018; 52 Supl 1: 8s.

15. LIMA CT, et al. Concurrent and constructo validity of the AUDIT in an urban Brazilian sample. Alcohol Alcohol. 2005; 40(6): 584-89

16. MÉNDEZ EB. Uma versão brasileira do AUDIT-Alcohol Use Disorders Identification Test. Dissertação de Mestrado, Universidade Federal de Pelotas, Pelotas, 1999.

17. MONTEIRO LZ, et al. Uso de tabaco e álcool entre acadêmicos da saúde. Rev. Bras. Promoç. Saúde [Internet], 2018 jan./mar; 31(1): 1-9.

18. PELICIOLI M, et al. Perfil do consumo de álcool e prática do beber pesado episódico entre universitários brasileiros da área da saúde. J. Bras. Psiquiatr [Internet], 2017; 66(3): 150-156. 
19. PINHEIRO MA, et al. Prevalência e fatores associados ao consumo de álcool e tabaco entre estudantes de medicina no nordeste do Brasil. Rev. Bras. Edu. Med. [Internet], 2017; 41(2): 231-250.

20. RIBEIRO RM. Estratégias protetoras e consequências negativas no consumo de bebidas alcoólicas por parte de estudantes universitários. Orientador: Joaquim Armando Ferreira. Dissertação (Mestrado em Psicologia da Educação, Desenvolvimento e Aconselhamento) - Faculdade de Psicologia e de Ciências da Educação. Universidade de Coimbra, Coimbra, 2018; $16 \mathrm{p}$.

21. SAWICKI WC, et al. Consumo de álcool, qualidade de vida, intervenção breve entre universitários de enfermagem. Rev. Bras. Enferm. [Internet], 2018; 71 Suppl 1: 547-555.

22. TRINDADE BPA et al. Uso de drogas entre estudantes universitários: uma perspectiva nacional. Rev. Med. Saude. Brasilia, 2018; 7(1):52-60.

23. WORLD HEALTH ORGANIZATION. Global Status Report on Alcohol and Health 2018 [Internet]. Suíça: WHO Library Cataloguing-in-Publication Data, 2018; 472 p.

24. ZEFERINO MT, et al. Consumo de drogas entre estudantes universitários: família, espiritualidade e entretenimento moderando a influência dos pares. Texto Contexto Enferm [Internet], 2015; 24(Esp): 125-35. 